\title{
Gerencia y Productividad en Sistemas Ganaderos de Doble Propósito
}

\author{
Nava Rosillón, Marbelis* \\ Urdaneta, Fátima** \\ Casanova, Angel ${ }^{\star \star *}$
}

\section{Resumen}

La ganadería de doble propósito se maneja con criterios gerenciales que poco se asocian a sus resultados productivos. El objetivo de esta investigación es explicar esta posible interrelación en 22 fincas situadas en el Municipio Sucre del Estado Zulia, seleccionadas por medio de un muestreo aleatorio simple. La información técnica y productiva se recolectó en el año 2004, utilizando un cuestionario y se procesó mediante técnicas estadísticas multivariantes. Se encontró que el proceso de gestión carece de lineamientos empresariales formalmente establecidos. Se definieron cuatro grupos de gestión, cuyos resultados reflejan que la mayoría de los productores están orientados a prácticas gerenciales de organización, ejecución y control. Los mejores resultados productivos y económicos están asociados a la gerencia tradicional basada en la experiencia de los productores, con énfasis en las funciones de ejecución, controly evaluación. Se concluye que los productores deben visualizar sus fincas como empresas, recibir capacitación para manejarlas con criterios gerenciales formales y así alcanzar altos resultados productivos y económicos que les permitan ser empresas más competitivas y rentables.

Palabras clave: Gerencia, Productividad, Grupos de Gestión, Ganadería de Doble Propósito, índice de gestión.

* $\quad$ Economista, LUZ. Magíster en Gerencia de Empresas, mención: Gerencia Financiera. Profesora Universitaria. Adm. de la Hacienda "Santa Rita", Municipio Sucre.

E-mail:marbejans@cantv.net .

** Ingeniero Agrónomo, LUZ. Magíster Scientiarum en Producción Animal. Profesora de la Facultad de Agronomía de la Universidad del Zulia. E-mail: fatimaurdanet@cantv.net

*** Ingeniero Agrónomo. Magíster Scientiarum en Estadística. Profesor emérito de la Facultad de Agronomía de la Universidad del Zulia. E-mail: angelcasa@cantv.net 


\title{
Management and Productivity of Dual Purpose Cattle Systems
}

\begin{abstract}
Dual purpose cattle are handled with managerial criteria that have little association with their productive results. The objective of this study is to explain this possible interrelation on 22 farms situated in the Sucre Municipality of the State of Zulia, selected by simple random sampling. Technical and productive information was collected in 2004 using a questionnaire and was processed using multivariate statistical techniques. It was found that the management process lacked formally established business guidelines. Four management groups were established, whose results reflected that most cattlemen are oriented toward managerial practices of organization, execution and control. The best productive and economic results are associated with traditional management, which is based on the cattlemen's experiences and emphasizes the functions of execution, control and evaluation. Conclusions are that cattlemen should visualize their farms as enterprises, receive training to manage them with formal management criteria and thereby, achieve high productive and economic results which will allow them to be more competitive and profitable businesses.
\end{abstract}

Key works: Management, productivity, management groups, dual purpose cattle, management index.

\section{Introducción}

En nuestros días, el sector agropecuario venezolano enfrenta importantes problemas referidos a la ausencia de infraestructura y de políticas gubernamentales que fomenten esta actividad, inadecuada organización del proceso productivo, escasa formación gerencial y carencia de criterios administrativos por parte de quienes manejan las empresas agropecuarias, aunado a un desigual desarrollo de sus unidades productivas; pues, este sector está conformado por fincas manejadas con criterio empresarial pero también conformado en una gran proporción, por fincas con baja productividad, que utilizan técnicas rudimentarias y son manejadas con criterio tradicionalista fundamentado en la experiencia de los productores, lo cual afecta la producción, productividad y rentabilidad.
Por lo tanto, el sector agropecuario venezolano requiere un mayor proceso de desarrollo mediante el cual sus unidades de producción sean manejadas con criterio empresarial para obtener mayor eficiencia y productividad de cada una de ellas y alcanzar la expansión del sector en su conjunto; para ello, es necesario conocer mejor la disponibilidad y combinación de los recursos y los procesos técnicos y administrativos aplicados en las fincas, de manera de lograr que los productores agropecuarios lleven una administración más eficiente de sus recursos que les permita obtener altos niveles de productividad, competitividad y rentabilidad.

Pues, en este sector existen muchos agricultores y ganaderos que no llevan contabilidad, ni administran eficientemente sus empresas, esto trae como consecuencia que aún cuando las fincas sean rentables, sus propietarios no están en 
Gerencia y Productividad en Sistemas Ganaderos de Doble Propósito

Nava Rosillón, Marbelis; Urdaneta, Fátima y Casanova, Angel

condiciones de conocer cuáles renglones de sus explotaciones generan mayor rendimiento, cuáles son insuficientes y cuáles generan pérdidas (Gómez,1994); por lo tanto, es necesario establecer un sistema de administración de fincas que permita conocer el desarrollo de la actividad productiva que se lleva a cabo en ellas.

En Venezuela, la ganadería de doble propósito constituye un sistema alternativo bastante viable para la producción de leche y carne en el país, por su adaptabilidad ecológica y factibilidad económica, y por ser el sistema de producción más eficiente para producir al mismo tiempo leche y carne aprovechando los recursos alimenticios forrajeros disponibles (González, 1992); por lo que se considera uno de los principales suplidores alimenticios de la población venezolana.

Aunado a esto, las nuevas tecnologías, los requerimientos de nuevos insumos, el manejo eficiente de recursos, los estados financieros ajustados por la inflación, la creación de presupuestos de ingresos y egresos con suficiente antelación, entre otros, han llevado a que la administración o gerencia de cualquier actividad productiva, especialmente en los sistemas de producción de doble propósito, adquiera una gran relevancia, aún cuando en el sector ganadero las funciones de gerencia relacionadas con la planificación, organización y dirección son puestas en práctica con frecuencia, pero se evidencian fallas en lo referente a la función de control y evaluación de resultados (Bermúdez y Aguirre, 1998), debido a la escasez de registros a nivel de fincas y, un inadecuado proceso contable, que dificulta el análisis del proceso productivo diario llevado a cabo en estos sistemas.
Específicamente, los ganaderos en el Municipio Sucre del Estado Zulia sufren esta problemática; y en la última década han enfrentado situaciones que se oponen al desarrollo del sector. Por una parte, han atravesado una aguda crisis de eficiencia, bajos niveles de productividad, que se reflejan en una disminución de sus niveles de rentabilidad, ausencia de políticas referidas a financiamiento, precios y comercialización de sus productos, y por otra parte, la liberalización del mercado y la poca intervención del Estado en la actividad agrícola han agravado el riesgo de disminución del aporte de esta región a la oferta nacional de leche y carne e incrementan los riesgos de crisis económica y social de la población.

En tal sentido, se presenta este trabajo de investigación, el cual tiene como objetivo principal explicar el comportamiento productivo de los sistemas de producción de ganadería de doble propósito ubicados en el Municipio Sucre del Estado Zulia en relación a la gestión llevada a cabo en ellos, describiendo las funciones de planificación, organización, ejecución, control y evaluación de dicha gestión, para tipificar gerencialmente las unidades de producción estudiadas e identificar los indicadores productivos que discriminan los grupos de gestión los cuales especifican las diferentes tipologías de fincas existentes en la zona.

Esto a su vez, permite conocer la eficiencia en el manejo de los recursos productivos disponibles en los negocios agropecuarios estudiados, de manera de orientar a los productores hacia la planificación, diseño de estrategias y control de su gestión, para mejorar la gestión productiva y gerencial en dichos sistemas, 
aplicando los correctivos adecuados para lograr un mejoramiento del proceso de producción y que el proceso de toma de decisiones sea más rápido y eficiente.

Además, la crisis que vive hoy en día el sector agropecuario evidencia la necesidad de realizar investigaciones en las unidades de producción y regiones agropecuarias para detectar las dificultades existentes en el manejo de los factores de producción agrícola y los resultados económicos de las fincas y así administrar las fincas como empresas, permitiendo a su vez medir los resultados económicos y financieros de su actividad, tomar decisiones en forma eficiente y promover cambios en la función referente al control y evaluación de los resultados financieros a través del manejo de registros en el ámbito agropecuario.

\section{Fundamentación Teórica}

Los cambios en el entorno exigen al gerente o productor poseer conocimientos de alto nivel que debe aprender formalmente, de manera que sea capaz de "adaptarse y tomar decisiones rápidas y oportunas para alcanzar el objetivo de productividad, rentabilidad y crecimiento" (Bermúdez y Aguirre, 1998:646) que se ha trazado para su actividad agrícola, la cual requiere imprescindiblemente la aplicación de criterios gerenciales, que junto a criterios técnicos y económicos permita una eficiente gestión de los factores que lleve a las empresas agropecuarias a obtener altos niveles de productividad y rentabilidad.

En efecto, la gestión empresarial está referida a las "prácticas directivas vinculadas con la forma de la autoridad, la toma de decisiones, los flujos de información, el control, la evaluación y el direccionamiento estratégico" (Pacheco et al., 2002: 5).

En otras palabras, el proceso de gestión se fundamenta en la práctica de acciones dirigidas a la resolución de los problemas que se presentan en el desarrollo de las operaciones diarias de cualquier empresa, con el propósito de cumplir con las metas y objetivos establecidos que permita un desempeño eficiente $y$ efectivo.

Ivancevich et al. (1997:11) afirma que "La gestión es un proceso que comprende determinadas funciones y actividades laborales que los gestores deben llevar a cabo a fin de lograr los objetivos de la empresa."

Así, la gestión constituye un proceso emprendido por una o más personas para coordinar las actividades laborales de otras personas, con la finalidad de lograr resultados de alta calidad, cuyo proceso se considera integrado por las funciones de gestión básicas, identificadas como: planificación, organización, control y evaluación vinculadas entre sí mediante la función de liderazgo.

La planificación es la función que determina los objetivos de una organización y establece las estrategias adecuadas para el logro de los mismos (Ivancevich et al., 1997). Es decir, es la función más elemental, pues, en esta primera fase se decide acerca de lo que se debe lograr y como conseguirlo, mediante la definición de los objetivos, procedimientos y políticas para alcanzar tales objetivos.

Además, la planificación permite que la organización identifique y aproveche las oportunidades externas y mi- 
Gerencia y Productividad en Sistemas Ganaderos de Doble Propósito Nava Rosillón, Marbelis; Urdaneta, Fátima y Casanova, Angel

nimice las consecuencias de las amenazas externas dado que es un proceso que permite identificar las oportunidades de mejoramiento en la operación de una empresa fundamentada en la técnica, así como también el establecimiento de planes para aprovechar dichas oportunidades (Gómez, 1994). En ese sentido, este proceso incluye la elaboración de una misión, pronósticos de las tendencias y los hechos futuros, el establecimiento de objetivos y la selección de las estrategias que se pondrán en práctica (David, 1997).

Por su parte, la organización es la función de gestión que comprende las tareas identificadas en el proceso de planificación y las asigna a determinados individuos y grupos dentro de la empresa de manera que los objetivos establecidos previamente en la planificación, sean alcanzados (Ivancevich et al., 1997). Esto significa que esta función facilita el cumplimiento de los objetivos mediante la agrupación y asignación de actividades y por la delegación de la autoridad.

"El propósito de la organización consiste en lograr un esfuerzo coordinado mediante la definición de una tarea y las relaciones de autoridad. Organizar significa determinar quién hace qué y quién depende de quién" (David, 1997:165).

En este sentido, la organización tiene como objetivo establecer las actividades necesarias para el buen funcionamiento de una empresa y asignar el recurso humano capacitado para su ejecución proporcionando la estructura formal para ello, de manera que este labore eficientemente.

En sí, la organización es la estructuración técnica de las relaciones entre las funciones, niveles y actividades de los recursos tanto humanos como materiales de una empresa (Gómez, 1994), cuyo propósito es lograr realizar los planes y objetivos establecidos en la planificación en forma eficiente.

En tanto, la función de ejecución se refiere a los esfuerzos dirigidos a dar forma al comportamiento humano; pues, se fundamenta en el liderazgo, el cual se define como el proceso de influir en los empleados de una organización para que se esfuercen en forma voluntaria y entusiasta en el cumplimiento de las metas establecidas (Koontz y O’Donnell, 1998). La planificación, organización y toma de decisiones no son efectivas hasta que el líder estimula el poder de la motivación en las personas y las dirige hacia el logro de los objetivos.

Así, se puede considerar que el liderazgo es un proceso mediante el cual se dirigen las actividades laborales de los empleados de una empresa y se influye en ellos mediante la comunicación, motivación y delegación, con la finalidad de alcanzar los resultados esperados según lo planificado.

En cuanto a la función control, David (1997:163) considera que: "Control se refiere a todas las actividades gerenciales que pretenden asegurar que los resultados reales sean consistentes con los resultados proyectados." En este sentido, controlar es la función de gestión que permite realizar evaluaciones de los resultados y tomar las medidas necesarias para minimizar las ineficiencias.

Guerra (1992:25) plantea que: "La función de control consiste en establecer estándares, comparar los resultados obtenidos con ellos y realizar los ajustes ne- 
cesarios para el logro de los objetivos trazados." Esto indica que los resultados obtenidos después de ejecutar el plan pueden desviarse de los resultados esperados dados ciertos factores externos; por ello se requiere un sistema de control que permita observar con frecuencia el plan y llevar un seguimiento de sus avances, de manera de medir los resultados con respecto a los objetivos establecidos.

Ahora bien, el análisis de la gestión de las empresas agropecuarias se basa en los resultados económicos y financieros de todo un ejercicio; es decir, se calcula y analiza cual fue el resultado económi$\mathrm{co}$, y se explica como se obtuvo haciendo una correcta asignación de costos e ingresos a cada una de las áreas y actividades productivas correspondientes a la composición del resultado global o patrimonial.

En este orden de ideas, es importante hacer referencia a los indicadores de gestión, los cuales constituyen instrumentos de medición de variables asociadas a las metas; pues el desempeño de toda empresa se mide y evalúa en términos de resultados, que se expresan en términos de indicadores de gestión.

Al respecto, Pacheco et al. (2002: 53) señala que "Un indicador numérico es una relación entre dos o más datos significativos, que tienen un nexo lógico entre ellos, y que proporcionan información sobre aspectos críticos o de importancia vital para la conducción de una empresa."

Estos indicadores están referidos a la relación entre las metas, los objetivos y los resultados y se definen como una unidad de medida gerencial o la expresión cuantitativa del desempeño de una organización, cuya magnitud puede determi- nar una desviación sobre la cual se tomarán acciones correctivas o preventivas.

Por otra parte, el manejo de las empresas agropecuarias en Venezuela se ve influenciado por el proceso de transformación que implica la globalización mundial por lo que estos negocios requieren ser gerenciados eficientemente, pués como toda empresa su objetivo principal es generar un beneficio.

La mayoría de estas fincas son manejadas intuitivamente y bajo la experiencia adquirida por los productores a través de los años, ya que estas son empresas familiares que se mantienen de una generación a otra y utilizan criterios técnicos y económicos tradicionales que ha permitido a estos productores perdurar en sus negocios, lo cual refleja el éxito de la actividad que realizan.

En los últimos años la situación ha cambiado, los productores buscan mejorar su desempeño y ser más eficientes, para ello se requiere una capacitación gerencial que les permita manejar sus fincas fundamentándose en criterios empresariales bien establecidos y así obtener resultados productivos y económicos satisfactorios.

En efecto, la práctica eficiente de las funciones gerenciales en los sistemas ganaderos lleva implícita una adecuada combinación en el uso de los recursos productivos, el logro de las metas y objetivos establecidos, un certero proceso de toma de decisiones, el buen funcionamiento operacional de la finca, así como también la detección de los problemas existentes en el desarrollo del proceso productivo, la aplicación de los correctivos más idóneos para solventarlos y de 
Gerencia y Productividad en Sistemas Ganaderos de Doble Propósito Nava Rosillón, Marbelis; Urdaneta, Fátima y Casanova, Angel

esta manera alcanzar los mejores resultados productivos y económicos.

En este sentido, los sistemas ganaderos de doble propósito, se definen como sistemas de ganadería que producen leche y carne a bajos costos, basados en el pastizaje fundamentalmente. Constituyen un sistema donde la vaca y el becerro conforman una unidad biológica natural de producción, que genera leche y carne en un período de tiempo determinado; con características específicas y uso de técnicas básicas relacionadas con el manejo de los pastizales, genética, reproducción y alimentación del rebaño, de manera de obtener ingresos a través de las actividades desarrolladas en ellos.

Morillo y Urdaneta (1998) definen el sistema de producción de doble propósito como un negocio que tiene como finalidad producir y vender leche y carne generalmente manejados por la propia familia, donde el rebaño es una mezcla de acebuados criollos y razas europeas que junto a la tierra constituyen el principal capital invertido.

En otras palabras, estos sistemas son considerados un negocio agrícola con la finalidad económica de producir y vender animales en pie y leche. Las diferentes fases de este negocio (cría, levante y ceba) pueden ocurrir en una o más fincas.

En Venezuela, la ganadería de doble propósito contribuye aproximadamente con el $90 \%$ de la producción nacional de leche que además representa el $60 \%$ del consumo total de leche (Ortega et al., 2007). Y está localizada principalmente en los Estados Zulia, Falcón, Mérida, Trujillo y Táchira, siendo el Estado Zulia la región que aporta el mayor porcentaje de estos rubros a nivel nacional.
Ahora bien, es importante referir lo relacionado con la productividad, pues, es la relación matemática entre la cantidad de producto elaborado y la cantidad de recursos utilizados en su producción expresada en términos físicos (productividad física); y por otra parte, establece la relación entre el valor bruto de la producción y uno o varios factores de la producción utilizados para obtenerlo, la cual es expresada en términos monetarios (productividad monetaria).

Para Pacheco et al. (2002: 60) "la productividad es la capacidad que tiene un sistema para integrar y combinar los recursos humanos, físicos y financieros que intervienen en la elaboración de un producto, con el propósito de utilizarlos económicamente".

La productividad permite determinar la eficiencia en la utilización de los factores de producción, de manera de definir la necesidad de alterar el uso de dichos factores para utilizarlos de la mejor forma posible, y así incrementar su rendimiento y su contribución en la producción; aunado a que se considera que los resultados productivos y económicos en las empresas ganaderas están asociados a un proceso gerencial eficiente y efectivo.

\section{Aspectos Metodológicos}

La presente investigación es de tipo explicativo, atraviesa un estadio descriptivo y otro comparativo; pues, busca explicar el comportamiento productivo de los sistemas de producción de ganadería de doble propósito en el Municipio Sucre del Estado Zulia en relación al proceso de gestión que se lleva a cabo en ellos, para lo cual se describen las funciones básicas 
de gestión referidas a la planificación, organización, ejecución, control y evaluación, se realiza una tipificación de las fincas estudiadas y se identifican los indicadores productivos que discriminan los grupos de gestión existentes en la zona (Cuadro 1). Este estudio se ubica dentro de un diseño no experimental, transeccional, de campo.

El universo estudiado estuvo conformado por 240 unidades de producción ubicadas en el Municipio Sucre según registros del Sistema Autónomo de Sanidad Animal (SASA). Este Municipio se localiza en la Zona Sur del Lago de Maracaibo, hacia el área sur oriental del Estado; de cuyo universo se seleccionó una muestra de 22 fincas mediante un muestreo aleatorio simple, la cual representa el $9,16 \%$ del total de la población investigada.

El proceso de recolección de datos se realizó tomando la información técnica-productiva del año 2004, utilizando la técnica de la encuesta y como instrumento un cuestionario ya desarrollado y disponible, cuya validación se realizó mediante la aplicación de la validez de contenido y de una prueba piloto que permitió modificar, ajustar y mejorar dicho instrumento. Es importante mencionar que el cuestionario utilizado está conformado por 51 ítems cerrados y 30 ítems abiertos y permitió conocer aspectos específicos de las fincas acerca de su ubicación, identificación del productor, funciones de gestión, indicadores técnicos, productivos, económicos y la tecnología utilizada en el proceso productivo de estas empresas.

Ahora bien, para el procesamiento de los datos se utilizaron técnicas estadísticas multivariantes, como el análisis de componentes principales, que permi- tió reducir la dimensionalidad de las variables y calcular el índice de gestión, el análisis cluster mediante el cual se agruparon las fincas estudiadas según la similitud en sus funciones de gestión conformando grupos de gestión definitivos, y el análisis discriminante que se fundamentó en seleccionar las variables que permiten diferenciar los grupos de gestión definidos. Estas variables incluyen indicadores productivos y económicos.

\section{Gestión de los Sistemas Ganaderos de Doble Propósito}

La gestión se considera como un proceso que comprende funciones fundamentales y actividades laborales que se deben realizar para lograr los objetivos establecidos (Ivancevich et al., 1997); por ello, con la finalidad de describir el proceso de gestión llevado a cabo en las fincas ganaderas de doble propósito en el Municipio Sucre, se identifican y analizan las funciones de planificación, organización, ejecución, control y evaluación que los productores agropecuarios realizan para manejar sus recursos eficientemente, fundamentados en su potencialidad.

Con respecto a la función de planificación en los sistemas estudiados, se determinó que la mayoría de los productores planifica, pero no plasma sus planes por escrito; no obstante, es fundamental que la planificación se realice por escrito, tanto para demostrar que existe como para su revisión (Gómez, 1994), de manera de verificar sí los resultados que se obtienen se ajustan a lo planificado (Tabla 1).

Además, la misión, visión, logros y objetivos no se encuentran definidos explícitamente como tal en estos sistemas 
Gerencia y Productividad en Sistemas Ganaderos de Doble Propósito

Nava Rosillón, Marbelis; Urdaneta, Fátima y Casanova, Angel

\section{Cuadro 1. Operacionalización de la Variable}

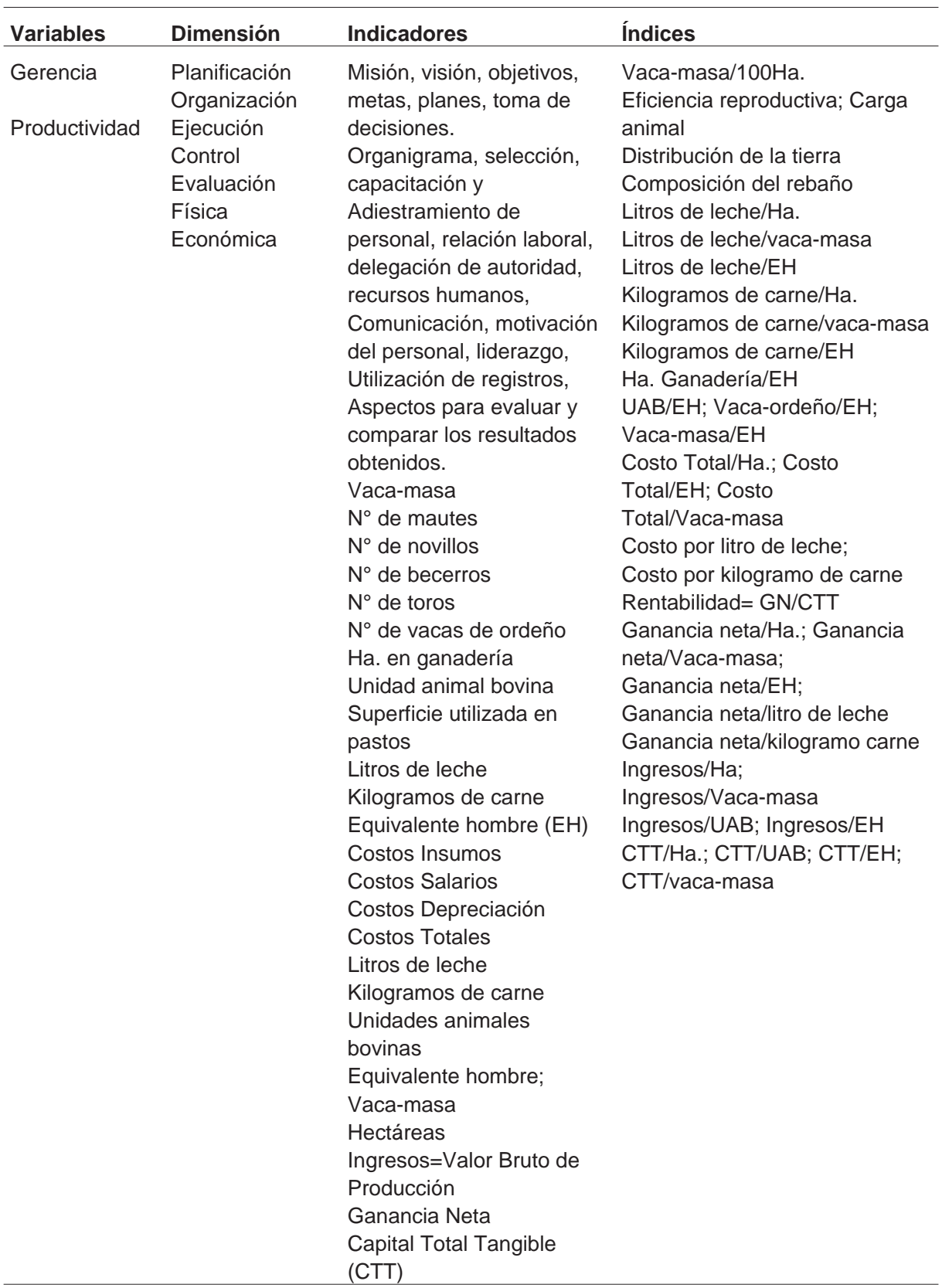

Fuente: Autores (2006). 
Tabla 1. Realización del proceso de planificación

\begin{tabular}{ccr}
\hline Categoría & $\mathbf{n}$ & $\%$ \\
\hline Planificación & & \\
Sí & 19 & 86,4 \\
No & 3 & 13,6 \\
Total & 22 & 100,00 \\
Planificación & & \\
por escrito. & 7 & 31,81 \\
Sí & 15 & 68,18 \\
No & 22 & 100,00 \\
Total & & \\
\hline
\end{tabular}

Fuente: Autores (2006).

ganaderos (Urdaneta et al., 1995). Sin embargo, se determinaron aspectos importantes que permiten establecer los fundamentos de los mismos en estos negocios agrícolas.

Los productores encuestados manejan un concepto reducido de la misión empresarial; ya que se basan principalmente en dos aspectos para definirla; vocación y tradición familiar; asimismo, la visión excluye ciertos elementos que pueden servir de estimulo y motivación para el mejoramiento de estas unidades de producción; debido a que estos empresarios solo toman en consideración la productividad y rentabilidad como criterios básicos para definir la visión, logros y objetivos de sus negocios, restándole importancia a otros criterios que también resultan fundamentales.

Las actividades operativas de los negocios agropecuarios, incluyen aspectos importantes como el uso de suplementos en la alimentación del ganado, lo cual está estrechamente relacionado con el manejo de pastizales; actividad que el productor debe planificar durante el año, dado que la alimentación animal constitu- ye un factor fundamental con mayor incidencia en la producción de leche y carne.

Asimismo, se determinó que el ganadero sucrense al momento de llevar a cabo una planificación se basa principalmente en el dinero que mantiene en caja, y las variaciones que sufren los precios de los productos que genera; así como también, que tan costosos pueden resultar los insumos agrícolas que requiere para cumplir con su proceso de producción.

En cuanto a la función de organización, se considera que las empresas ganaderas estudiadas mantienen una estructura autocrática con líneas de mando jerárquico o vertical orientado a realizar las actividades gerenciales, técnicas y económicas en función de los objetivos establecidos, pero no existe departamentalización alguna, sino que en la mayor parte de estas empresas agropecuarias, se presenta una estructura en la cual el productor representa la principal figura jerárquica y cumple las funciones técnicas, gerenciales y económicas; es decir, es quien toma las decisiones con el apoyo técnico de un médico veterinario o ingeniero agrónomo, y delega autoridad y responsabilidad en el encargado, quien aparece como jefe de las actividades operativas de las fincas, pues supervisa y controla al personal obrero, con respecto a lo cual se encontraron cuatro diferentes niveles de autoridad en estos sistemas de producción ganaderos, denominados de la siguiente manera:

Nivel A: Define una relación lineal donde el productor toma las decisiones gerenciales y técnicas y realiza labores de supervisión y control sobre los obreros, cuyo nivel se presenta en el 18,18\% de las explotaciones agropecuarias. 
Gerencia y Productividad en Sistemas Ganaderos de Doble Propósito

Nava Rosillón, Marbelis; Urdaneta, Fátima y Casanova, Angel

Nivel B: Define una estructura jerárquica de mando donde el productor asume las funciones gerenciales, técnicas y económicas, y delega en la figura del encargado, quien a su vez delega funciones de ejecución y supervisión sobre el resto de los trabajadores, cuyo nivel existe en el $31,81 \%$ de los sistemas ganaderos.

Nivel C: Es un nivel donde el productor constituye la principal figura de la estructura jerárquica de mando que cumple con las funciones gerenciales y económicas, dispone de la asesoría técnica de un médico veterinario o de un ingeniero agrónomo privado, y encarga funciones de ejecución y supervisión en el encargado, y este delega en el resto del personal; este nivel se encontró en la mayoría de las fincas alcanzando una proporción del 36,36\%.

Nivel D: Constituye un nivel en el cual el productor aparece como la principal estructura jerárquica de mando que toma las decisiones económicas, gerenciales y técnicas, recibe apoyo técnico de un médico veterinario o de un ingeniero agrónomo y asesorías administrativas de un contador público. Además delega las funciones de ejecución y supervisión en el encargado y éste en los obreros; cuyo nivel alcanza la menor proporción con respecto a los niveles anteriores, ya que representa $13,63 \%$ del total de las unidades de producción analizadas.

Por otra parte, la función organización presenta deficiencia en el manejo de los recursos humanos, al establecer la relación laboral mediante un contrato oral y por la ausencia de capacitación y entrenamiento al personal; aunado al bajo nivel educativo que tiene la fuerza de traba- jo que opera en estos negocios agrícolas; por lo tanto, es imprescindible ofrecer facilidades, apoyo y oportunidades de educación y adiestramiento al personal, a fin de lograr un buen funcionamiento operativo de las fincas que conlleve al incremento de los resultados de la actividad agropecuaria.

No obstante, en la Tabla 2 se muestran los fundamentos de la función de ejecución, en la cual las cifras de las actividades que los productores-gerentes encuestados deciden en equipo señalan que las actividades técnico-operativas como el manejo de pastizales, control sanitario, alimentación del rebaño, control de malezas, contratación y selección del personal, descarte y selección del rebaño, manejo sanitario, control reproductivo, suplementación para el ganado, ordeño, compra y venta de animales y venta de productos, presentan valores mayores al $80 \%$; actividades como el mantenimiento de maquinarias, equipos, construcciones e instalaciones, se ubican con un valor de 59,09\%; y sólo el control de plagas presenta la proporción más baja $(13,63 \%)$. Esto refleja que los productores-gerentes comparten su poder para la toma de decisiones con el resto de las personas involucradas en el proceso de ejecución, tomando como base la participación, cooperación y solidaridad del equipo de trabajo.

De allí, la función de ejecución en los sistemas de doble propósito estudiados resulta satisfactoria; en lo cual influye que la comunicación se establece en forma oral permitiendo transmitir la información u órdenes y recibir una respuesta inmediata directamente al personal indicado; también contribuye el hecho que los 


\section{Tabla 2. Fundamentos del Proceso de Ejecución}

\begin{tabular}{lcc}
\hline Categoría & $\mathbf{n}$ & $\%$ \\
\hline Comunicación con el Personal. & & \\
Administrador. & 5 & 22,72 \\
Encargado. & 13 & 59,09 \\
Obreros. & 11 & 50,00 \\
Todo el personal. & 4 & 18,18 \\
Hijos. & 1 & 4,54 \\
Motivación del Personal. & 5 & 22,72 \\
Otorga dinero extra además del salario. & 2 & 9,10 \\
Mayor tiempo libre. & 2 & 9,10 \\
Oportunidad de educación personal. & 12 & 54,54 \\
Incentivos materiales. & 14 & 63,63 \\
Facilidad de préstamos. & 21 & 95,45 \\
Actividades que decide en equipo. & 21 & 95,45 \\
Manejo de pastizales. & 19 & 86,36 \\
Control sanitario. & 18 & 81,81 \\
Alimentación. & 21 & 95,45 \\
Control de malezas. & 21 & 95,45 \\
Contratación y selección de personal. & 20 & 90,90 \\
Descarte y selección del rebaño. & 13 & 59,09 \\
Manejo sanitario. & 21 & 95,45 \\
Mantenimiento de maquinarias. & 19 & 86,36 \\
Control reproductivo. & 22 & 100,00 \\
Suplementación para el ganado. & 3 & 13,63 \\
Ordeño. & 13 & 59,09 \\
Control de plagas. & 19 & 86,36 \\
Mantenimiento de construcciones e inst. & 19 & 86,36 \\
Compra y venta de animales. & & \\
Venta de productos. & & \\
\hline Fuente: Autores (2006) & & \\
\hline
\end{tabular}

Fuente: Autores (2006)

productores-gerentes sean capaces de ejercer influencia en su personal, basados en la autoridad inherente a su posición, mediante una motivación monetaria a su fuerza de trabajo, a fin de incrementar su rendimiento para alcanzar las metas y objetivos establecidos; aunado a un liderazgo autocrático pero participativo que coadyuve a una toma de decisiones más acertada; pues, los productores sucrenses son líderes que consultan al personal respecto a sus acciones y decisiones, estimulando su participación y per- mitiendo su apoyo en el establecimiento de metas y de los medios para alcanzarlas; y a su vez, mantienen un liderazgo autocrático mediante el cual imponen y esperan cumplimiento de las órdenes que imparten como figura jerárquica principal (Koontz y O’Donnell, 1998).

Ahora bien, para cumplir con la función de control a cabalidad, se requiere de un sistema de control efectivo, que permita el logro de las metas organizacionales; es por ello, que en las empresas agropecuarias el sistema de control se 
Gerencia y Productividad en Sistemas Ganaderos de Doble Propósito Nava Rosillón, Marbelis; Urdaneta, Fátima y Casanova, Angel

fundamenta en el uso de registros que faciliten observar el plan y realizar un seguimiento del progreso del mismo para medir los resultados del proceso productivo con respecto a los objetivos establecidos (Guerra, 1992).

En relación al sistema de registros utilizados por los productores-gerentes para el control de su proceso productivo; se determinó el uso de cuatro tipos de registros: registros productivos (54,54\%), reproductivos $(68,18 \%)$, operativos (59, $09 \%$ y contables $(36,36 \%)$ siendo estos últimos los menos utilizados (Gráfico 1); los cuales, según información de los productores son llevados en forma manual mediante el uso de una agenda o cuaderno donde se anota toda la información.

Una vez que la información se asienta en los registros, los productoresgerentes deben proceder a la evaluación de los resultados de su actividad; de manera tal, que determinen la eficiencia de su gestión; es decir, sí está cumpliendo con las metas fijadas, sí utilizan en forma efectiva los recursos, métodos y como operan sus fincas; para lo cual, la mayoría de los productores del Municipio Sucre se fundamentan en criterios como los resultados

\section{Gráfico 1. Tipos de Registros}

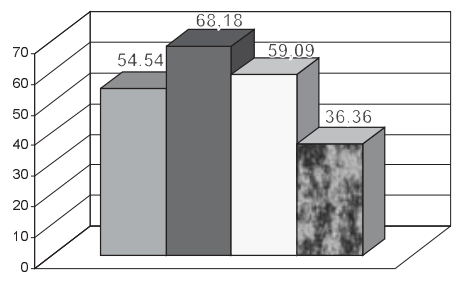

QProductivos Reproductivos 口Operativos GContables

Fuente: Autores (2006). económicos (50\%) y la productividad del negocio (50\%); y muy pocos productores toman en cuenta el logro de metas y objetivos $(13,63 \%)$, crecimiento de la finca $(22,72 \%)$ y la rentabilidad $(13,63 \%)$. Además, un número considerable de productores evalúan sus resultados semanalmente $(40,90 \%)$, otros lo hacen una vez al mes $(31,81 \%)$, algunos anualmente $(27,27 \%)$, y muy pocos manifestaron no evaluar (13,63\%), (Tabla 3).

Estos resultados sugieren que la gran mayoría de los empresarios del campo están sensibilizados para cumplir con la evaluación de su actividad, dada la importancia de esta función de gestión, que permite conocer cómo es el desempeño real de las fincas, aún cuando sólo evalúan básicamente los resultados productivos y económicos, restándole importancia a la rentabilidad y crecimiento de la finca, así como a la consecución de metas y objetivos establecidos.

Aunado a esto, se hace necesario comparar los resultados obtenidos; pues, ello determina el grado de variación entre el desempeño real y el deber ser (Robbins, 1996). Por ende, se indagó acerca de los criterios en los cuales se apoyan los productores Sucrenses para comparar sus resultados; de esto se determinó que un número considerable de ellos no relaciona sus resultados con parámetros estándares $(68,18 \%)$, otros confrontan los resultados actuales contra los obtenidos en años anteriores (31, $81 \%)$; y son muy pocos quienes se basan en su propia experiencia $(18,18 \%)$ y en los resultados de la finca piloto de la zona $(13,63 \%)$, (Tabla 4). Estas cifras sugieren que los productores encuestados no consideran importante este paso para el con- 
Tabla 3. Evaluación y Comparación de Resultados

\begin{tabular}{lcc}
\multicolumn{1}{c}{ Categoría } & N & $\%$ \\
\hline Aspectos considerados para evaluar. & & \\
Logro de metas y objetivos. & 3 & 13,63 \\
Crecimiento y consolidación de la finca. & 5 & 22,72 \\
Rentabilidad. & 3 & 13,63 \\
Resultados económicos. & 11 & 50,00 \\
Productividad del negocio. & 11 & 50,00 \\
Frecuencia para evaluar. & 3 & 13,63 \\
No evalúa. & 9 & \\
Semanal. & 7 & 40,90 \\
Mensual. & 6 & 31,81 \\
Anual & & 27,27 \\
Comparación de resultados. & 7 & 31,81 \\
Actividad de la finca en años anteriores. & 4 & 18,18 \\
Experiencia del productor. & 3 & 13,63 \\
Finca piloto de la zona. & 11 & 68,18 \\
No compara resultados con parámetros. & & \\
\hline
\end{tabular}

Fuente: Autores (2006).

trol y gestión de sus fincas; aún cuando comparar contribuye a determinar sí se opera bien o sí algo no funciona favorablemente.

Lo expuesto anteriormente muestra una función de control en las fincas del Municipio Sucre caracterizada por la utilización de un sistema de registros que permite asentar toda la información requerida para conocer como funcionan las fincas, aún cuando éste se lleva en forma manual y presenta deficiencia en la evaluación y comparación de los resultados de su actividad; ya que los productores se fundamentan principalmente en los resultados productivos y económicos sin tomar en cuenta otros criterios importantes como rentabilidad, crecimiento, logros y objetivos; y consideran poco necesario comparar sus resultados; entonces, esto conlleva a que la función control no se cumpla en forma efectiva en los sistemas ganaderos de doble propósito estudiados.

\section{Tipificación Gerencial de los Sistemas Producción de Ganadería de Doble Propósito}

Para la construcción del índice de gestión, se procedió a agrupar los sistemas de producción involucrados en esta investigación según la similitud en sus funciones de gestión, mediante el análisis de componentes principales sobre las cinco funciones del proceso de gestión llevado a cabo en estos sistemas como son: planificación, organización, ejecución, control y evaluación.

Ahora, cada función del proceso de gestión repercute en la siguiente, produciéndose un ciclo, y considerándose componentes mutuamente dependientes que no pueden estudiarse en forma aislada, ya que "estas fases cuando son consideradas como un todo forman el proceso gerencial; si se consideran en forma aislada, los objetivos no se cumplirian a ca- 
Gerencia y Productividad en Sistemas Ganaderos de Doble Propósito Nava Rosillón, Marbelis; Urdaneta, Fátima y Casanova, Angel

balidad y afectarían la producción y productividad de la finca" (Bermúdez y Aguirre, 1998:648). Esta situación sugiere que estos componentes interactúan en una misma dirección permitiendo el análisis de correlación de los mismos.

La Tabla 4 presenta los eigenvalores de la matriz de correlación, los cuales aportan información acerca de la varianza contenida en los dos componentes principales, cuyos componentes explican el $77,52 \%$ de la variación total.

El primer componente principal explica el $58,40 \%$ de la variación total; mientras que, el segundo componente explica el $19,12 \%$. Incluyendo sólo aquellos eigenvalores aproximados al promedio (superiores que 1), se seleccionaron los dos primeros componentes principales, que según Quevedo (1991) y Demey (1994), citados por Peña (1995:46), "en el análisis de los componentes principales, cada componente es el resultado de una combinación lineal de las variables en donde cada una de ellas tiene una ponderación diferente, en proporción a las magnitudes de cada elemento que conforma el eigenvector respectivo."

El primer componente principal, es el que presenta la varianza más alta y por ello, la capacidad explicatoria de los datos. En este componente se observan valores positivos en proporciones similares

Tabla 4. Eigenvalores de la matriz de correlación

\begin{tabular}{cccc}
\hline & Eigenvalor & Proporción & $\begin{array}{r}\text { Proporción } \\
\text { acumulada }\end{array}$ \\
\hline CP1 & 2.9197 & 0.5840 & 0.5840 \\
CP2 & 0.9561 & 0.1912 & 0.7752 \\
\hline
\end{tabular}

CP1: Componente Principal 1.

CP2: Componente Principal 2.

Fuente: Autores (2006). a aquellas funciones que en conjunto reflejan el proceso de gestión de las fincas.

Estos resultados se presentan en la Tabla 5, y se pueden interpretar en el sentido de que las magnitudes de este componente están asociadas positivamente a las funciones básicas del proceso de gestión: planificación, organización, ejecución, control y evaluación. Esto señala que el índice de gestión dado por el primer componente principal asocia valores altos con sus pares en todas las funciones componentes, y viceversa.

Con respecto al segundo componente, que explica una fracción menor de la varianza total, se observa que las funciones con más alto coeficiente son organización y control, que presentan valores positivos de 0,8259 y 0,2384 respectivamente; y planificación, ejecución y evaluación con valores negativos de 0,2819, 0,4219 y 0,0578 respectivamente ( $\mathrm{Ta}$ bla 5). Este componente indica que algunos sistemas productivos presentan valores altos de las funciones organización y control, pero valores bajos de las funciones planificación, ejecución y evaluación.

Por otra parte, mediante el Análisis Cluster se logró la agrupación definitiva

Tabla 5. Componentes Principales y sus Eigenvectores

\begin{tabular}{lcc}
\hline Funciones & CP1 & CP2 \\
\hline Planificación & 0.5126 & -0.2819 \\
Organización & 0.3004 & 0.8259 \\
Ejecución & 0.4530 & -0.4219 \\
Control & 0.4788 & 0.2384 \\
Evaluación & 0.4608 & -0.0578 \\
\hline
\end{tabular}

CP1: Componente Principal 1.

CP2: Componente Principal 2.

Fuente: Autores (2006). 
de las fincas formando grupos disjuntos a partir de las distancias euclidianas presentes entre ellos, denominados grupos de gestión. El Gráfico 2, muestra la dispersión de los sistemas ganaderos estudiados, proyectando una panorámica de la agrupación de los mismos. Asimismo, se tiene la Tabla 6 , que presenta los valores promedios de los grupos de gestión correspondientes a cada función del proceso de gestión.

Grupo 1: Este grupo está constituido por el $39,13 \%$ de las fincas que conforman la muestra, ubicadas entre el primer, segundo y tercer cuadrante del gráfico de dispersión (Gráfico 2). Se observa en la Tabla 6, que estas empresas agropecuarias presentan los valores promedios más altos para las funciones organización $(0,3763)$, ejecución $(0,4171)$ y control $(0,4444)$ y los valores promedios más bajos para las funciones planificación $(0,2422) y$ evaluación $(0,1074)$; lo cual re- fleja que son productores con prácticas poco vinculadas a la planificación de las actividades productivas y evaluación de sus resultados, aún cuando están identificados con prácticas orientadas hacia la organización del trabajo, conducción del personal y control de los procesos ejecutados en la actividad productiva.

Grupo 2: Grupo constituido por el $17,4 \%$ de los sistemas de producción estudiados. Se observa en el Gráfico 2 que se ubican entre el primer, tercer y cuarto cuadrante. Este grupo presenta valores promedios muy próximos entre sí para las funciones del proceso de gestión; ya que según las cifras de la Tabla 6 , los índices de gestión más elevados corresponden a las funciones ejecución $(0,3531)$, control $(0,3125)$ y evaluación $(0,3583)$, y los más bajos alcanzan valores muy similares de 0,2977 y 0,2901 para las funciones planificación y organización, respectivamente. Estos resultados sugieren la inclinación

\section{Gráfico 2. Diagrama de Dispersión de los Sistemas de Producción de Doble Propósito}

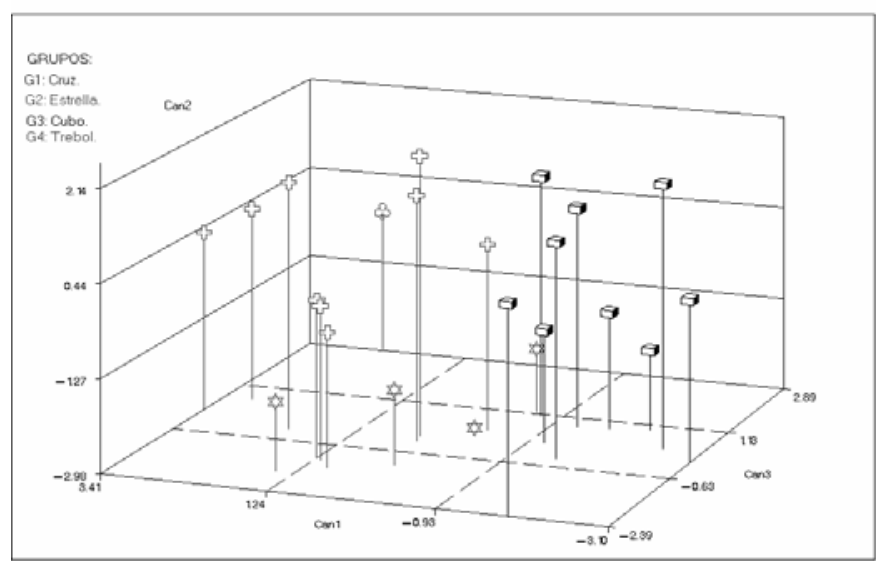

Fuente: Autores (2006). 
Gerencia y Productividad en Sistemas Ganaderos de Doble Propósito Nava Rosillón, Marbelis; Urdaneta, Fátima y Casanova, Angel

de los productores de estas cuatro fincas hacia una práctica proporcional de las funciones de gestión, manteniendo criterios técnicos y gerenciales tradicionales basados en su propia experiencia.

Grupo 3: Se encuentra constituido por el $39,13 \%$ de las fincas analizadas, las cuales se ubican entre el primer y cuarto cuadrante del gráfico de dispersión (Gráfico 2). Alcanzan valores promedios altos que reflejan eficiencia en la realización de las funciones del proceso de gestión; los más altos índices corresponden a las funciones de ejecución $(0,6013)$ y control $(0,666)$; y los valores más bajos pero muy próximos entre ellos corresponden a las funciones planificación $(0,4237)$, organización $(0,3353)$ y evaluación $(0,3555)$ (Tabla 6). Estas cifras indican la tendencia de estos productores hacia el manejo de sus fincas con criterio empresarial, interrelacionando las funciones gerenciales con el fin de alcanzar las metas establecidas, partiendo de la planificación de las actividades productivas e insumos disponibles, de manera de lograr una gestión eficiente de su negocio.

Grupo 4: Es un grupo constituido por un sistema de producción, representando el 4,34\% del total de fincas estudiadas. Éste se ubica en el segundo cua- drante del gráfico de dispersión (Gráfico 2), y presenta valores promedios muy altos que sugieren mayor eficiencia en la puesta en práctica de las funciones del proceso de gestión que el grupo anterior. Según indica la Tabla 6, los valores promedios más altos corresponden a las funciones organización y control con cifras de 0,6696 y 1.000 respectivamente; y las funciones planificación, ejecución y evaluación alcanzan valores promedios muy próximos, iguales a 0,4254, 0,4875 y 0,5333 respectivamente. Este productor se identificó con el hecho de visualizar su finca como un negocio orientando sus esfuerzos hacia una toma de decisiones adecuada con los recursos humanos e insumos disponibles, utilizando tecnologías modernas, capacitación del personal como fuente de nuevos conocimientos, otorgando importancia a la experiencia y motivación de los trabajadores e inclinándose hacia la organización y control de los procesos llevados a cabo en la actividad agropecuaria que dirige.

\section{Indicadores Productivos Discriminantes de los Grupos de Gestión}

Los indicadores de productividad seleccionados son litros de leche por

Tabla 6. Valores Promedio de los Grupos de Gestión

\begin{tabular}{lcccc}
\hline Funciones/Grupos & Grupo 1 & Grupo 2 & Grupo 3 & Grupo 4 \\
\hline $\mathrm{n}$ & 9 & 4 & 9 & 1 \\
Planificación & 0.2422 & 0.2977 & 0.4237 & 0.4254 \\
Organización & 0.3763 & 0.2901 & 0.3353 & 0.6696 \\
Ejecución & 0.4171 & 0.3531 & 0.6013 & 0.4875 \\
Control & 0.4444 & 0.3125 & 0.6666 & 1.0000 \\
Evaluación & 0.1074 & 0.3583 & 0.3555 & 0.5333 \\
\hline
\end{tabular}

Fuente: Autores (2006). 
vaca de ordeño al día, litros de leche por hectárea, kilogramos de carne por hectárea y carga animal, y entre los indicadores económicos están costo total por litro de leche, costo total por hectárea, ingreso total por hectárea y ganancia neta por hectárea. El análisis discriminante no seleccionó variables como la rentabilidad, ya que en el Grupo 4 el valor de ganancia neta es negativo; y por lo tanto, esta no puede ser calculada.

En la Tabla 7 se presentan los valores promedios de las variables discriminantes en relación a los grupos de gestión presentes en el Municipio Sucre, que permiten agrupar las diferentes tipologías de fincas existentes en la zona.

Grupo 1: Los negocios identificados en este grupo de gestión presentaron la menor producción de leche por vaca de ordeño al día con un valor de 5,94 litros, similar al reportado por Peña (1995), de 5,87 litros/VO/día para un grupo de fincas clasificadas según el nivel gerencial en grupo bajo; y muy próximo al valor de producción de 5,53 litros/VO/día reportado por Rincón (2004) al evaluar un grupo de fincas en el Municipio Catatumbo clasificadas según la intensidad del capital; cuyos resultados sugieren que esta respuesta productiva se debe a la presencia de animales hembras de bajo nivel productivo en el rebaño de producción.

En relación a la productividad por hectárea, este grupo alcanza una producción de 1.063 litros de leche y 167,48 kilogramos de carne, los cuales se aproximan a los reportados en la investigación de Rincón (2004) para un grupo de fincas con valores de 1.431,51 litros de leche y

Tabla 7. Valores de variables discriminantes por grupo de gestión

\begin{tabular}{lcccccc}
\hline $\begin{array}{l}\text { Variables } \\
\text { Discriminantes }\end{array}$ & Unidad & $\begin{array}{c}\text { Grupo } 1 \\
\mathbf{n = 8} \\
\text { Media }\end{array}$ & $\begin{array}{c}\text { Grupo 2 } \\
\mathbf{n = 2} \\
\text { Media }\end{array}$ & $\begin{array}{c}\text { Grupo 3 } \\
\mathbf{n = 9} \\
\text { Media }\end{array}$ & $\begin{array}{c}\text { Grupo 4 } \\
\mathbf{n = 1} \\
\text { Media }\end{array}$ & $\begin{array}{c}\text { Total. } \\
\mathbf{n = 2 0} \\
\text { Media }\end{array}$ \\
\hline $\begin{array}{l}\text { Indicadores de } \\
\text { Productividad }\end{array}$ & & & & & & \\
LTVODIA & Lt/Vaca/día & 5,94 & 8,74 & 6,42 & 6,66 & 6,47 \\
LECHEHa. & Lt/ha. & 1.063 & 2.637 & 954,08 & 1.678 & 1.202 \\
CARNEHa. & Kg./ha. & 167,48 & 197,92 & 141,01 & 128,36 & 156,66 \\
CARGA & UAB/ha. & 1,41 & 1,89 & 1,27 & 1,53 & 1,40 \\
Indicadores & & & & & & \\
Económicos & & & & & & \\
CTLT. & Bs./Lt. & 614,20 & 137,61 & 351,45 & 400,01 & 437,60 \\
CTHa. & Bs./ha. & 469.330 & 365.393 & 326.775 & 671.289 & 404.884 \\
INGHa. & Bs./ha. & 564.015 & 1.260 .403 & 569.188 & 622.415 & 638.902 \\
GNHa. & Bs/ha & 94.686 & 895.010 & 242.413 & -48.874 & 234.018 \\
\hline N N & & & & & & \\
\hline
\end{tabular}

$\mathrm{N}$ : Número de fincas.

LTVODIA: Litros de Leche por Vaca de Ordeño al día.

Ha.: Hectárea.

CT: Costo Total.

Fuente: Autores (2006) 
Gerencia y Productividad en Sistemas Ganaderos de Doble Propósito Nava Rosillón, Marbelis; Urdaneta, Fátima y Casanova, Angel

163,81 kilogramos de UAB/ha.; valores comprendidos entre 1,2 UAB/ha. y 1,8 UAB/ha., reportados por Urdaneta et al. (1995) para fincas del Sur del Lago de Maracaibo, lo cual sugiere ser favorable.

Lo expuesto anteriormente hace suponer que en este grupo de fincas no se lleva un manejo eficiente de los pastos y la suplementación alimenticia no es suficiente para suplir los requerimientos nutricionales de los animales; constituyendo la rotación de potreros, fertilización; entre otros; problemas característicos en cuanto a la alimentación del ganado en la Zona Sur del Lago de Maracaibo.

De allí, que los valores de productividad que arroja este grupo se pueden incrementar mediante un manejo eficiente de potreros, una efectiva suplementación mineral y un mayor uso de alimentos concentrados o proteicos.

Los resultados económicos para este grupo, indican el más alto costo total por litro de leche que alcanza un valor de $614,20 \mathrm{Bs}$./Lt, reflejando que en este grupo se incurre en un mayor costo para producir cada litro de leche en relación al resto de los grupos. El costo total por hectárea es de Bs.469.330, muy próximo al costo total de $476.930 \mathrm{Bs}$./ha. obtenido para un grupo de fincas estudiadas por Rincón (2004). Además, este grupo generó el menor ingreso por hectárea, igual a Bs.564.015, y una ganancia neta de Bs. 94.686 por hectárea de tierra.

No obstante, el manejo de los recursos y su resultado productivo está directamente relacionado con las funciones que componen el índice de gestión, donde las funciones planificación y evaluación presentan los índices más bajos (Tabla 6); resaltando con el valor más alto la función control $(0,4444)$, la cual no es suficientemente efectiva sino está acompañada de planes, disposición para la utilización de nuevas tecnologías, capacitación, motivación del personal, etc.

Estos resultados hacen suponer que los productores identificados en este grupo realizan actividades de planificación que no están vinculadas a la función de ejecución del proceso de gestión; por ello, estos productores, para manejar sus empresas agropecuarias en forma eficiente deben examinar los recursos disponibles, las posibilidades técnicas para su utilización y combinación, de manera de lograr un uso más efectivo de los mismos.

Grupo 2: En este grupo se encuentran aquellos negocios de doble propósito que presentan el mejor comportamiento productivo, ya que arroja los valores promedios más altos de la muestra. Alcanzan una producción de 8,74 litros de leche por vaca de ordeño al día y 2.637 litros de leche por hectárea, valores más altos a los reportados por Rincón (2004) para un grupo de finca en el Municipio $\mathrm{Ca}$ tatumbo que presenta los mayores resultados productivos de la muestra con valores de 7,81 Lt/NO/día y 1,796,6 Lt/ha..

Sin embargo, la producción promedio de carne para este grupo es de 197,92 $\mathrm{Kgs} / \mathrm{ha}$., resultando menor que la producción promedio de carne reportada por Rincón (2004) para un grupo de fincas similar, cuyo valor es de 220,23 Kgs./ha.; y se ubica entre los valores reportados por Urdaneta et al (1995) para fincas de la Zona Sur del Lago de Maracaibo, cuyos valores varían desde $89,4 \mathrm{Kgs} / \mathrm{ha}$. a 225 $\mathrm{Kgs} / \mathrm{ha}$. También, este grupo de fincas muestra la mayor carga animal de 1,89 
UAB/ha., similar a la reportada por Rincón (2004) con un valor de 1,88 UAB/ha..

Estos resultados indican que en este grupo de fincas se parte de prácticas agronómicas efectivas para garantizar el suministro de pasto al rebaño, como un efectivo control químico y manual de malezas, fertilización, una adecuada rotación de potreros, un adecuado manejo de la suplementación alimenticia recibida por el rebaño de producción y la presencia de animales con un alto potencial lechero, ya que el valor de estas variables aumenta en la medida que se posea animales hembras productivas con mayor calidad genética, lo cual incide en una mayor producción y productividad de las empresas agropecuarias.

Los resultados de productividad indican un comportamiento económico favorable para estos negocios ganaderos, presentando el menor costo total por litro de leche de Bs. 137,61, con costo de Bs. 365.393 por hectárea, y el más alto ingreso por hectárea de Bs. 1.260.403, y la mayor ganancia neta por hectárea de Bs. 895.010; los cuales son producto del manejo llevado a cabo en estas fincas, y reflejan una adecuada combinación de los recursos basados fundamentalmente en la ejecución, control y evaluación de la actividad de sus negocios, cuyas funciones de gestión alcanzan los índices más altos para este grupo de fincas (Tabla 7).

Los productores-gerentes identificados en este grupo tienden a una gestión con funciones llevadas a cabo en forma proporcionada, ya que los indicadores productivos y económicos obtenidos sugieren una planificación establecida por los productores a fin de mantener un rebaño eficiente, tanto en la producción de le- che como en la producción de carne, de manera que las actividades que se ejecuten constituyan los medios para una aplicación eficiente y coordinada del trabajo.

Aunado a esto, el control y evaluación que estos productores-gerentes efectúan de los resultados de su actividad permiten determinar cómo están actuando y como se encuentra su finca, cuándo se desvían de sus objetivos y cómo ajustarse de nuevo a ellos, detectando aciertos y fallas, y así aplicar las medidas correctivas requeridas para solventar los problemas que puedan obstaculizar el desarrollo de su potencial productivo lo cual permite llevar una gestión adecuada de sus negocios.

Grupo 3: Los indicadores de productividad arrojados por este grupo de fincas presentaron valores promedios de 6,42 litros de leche por vaca de ordeño al día, superior al reportado por Peña (1995) para un grupo de finca con nivel gerencial alto, cuyo valor es de 6,12 Lt/VO/día. La producción promedio de leche por hectárea es la más baja de la muestra, sólo alcanza 954,08 litros, superior a la reportada por Rincón (2004) para un grupo de fincas con una producción de 779,9 Lt/ha.. Mientras que, la producción promedio de carne para este grupo, es una de las más baja de la muestra, igual a 141,01 kilogramos por hectárea; y sustenta una carga animal de 1,27 UAB/ha. Este comportamiento productivo resulta satisfactorio para estas empresas, aún cuando estos resultados sugieren un mediano potencial lechero de animales hembras, baja carga animal y un inadecuado manejo de potreros.

Este grupo presentó resultados económicos que indican un costo de Bs. 
Gerencia y Productividad en Sistemas Ganaderos de Doble Propósito

Nava Rosillón, Marbelis; Urdaneta, Fátima y Casanova, Angel

351,45 por litro de leche, el menor costo por hectárea de Bs. 326.775 y un ingreso por hectárea muy bajo de Bs. 569.188, y una ganancia neta de Bs. 242.413 por hectárea de superficie. Estos bajos valores de ganancia, ingreso y costo por hectárea sugieren poca inversión en insumos tecnológicos requeridos para un manejo animal adecuado en estos negocios ganaderos. Además, estos resultados se encuentran directamente vinculados al manejo gerencial llevado a cabo por estos productores-gerentes, quienes hacen énfasis en la ejecución y control de las actividades del proceso productivo, funciones mayormente destacadas en este grupo (Tabla 7).

De allí, se estima que estos productores manejan sus fincas mediante la fijación de metas y objetivos, elaboración de planes para las actividades del proceso productivo, motivación del recurso humano, efectuando un control y evaluación de los resultados de su actividad, lo cual parece no es suficientemente adecuado, dados los bajos resultados económicos; por ello, estos ganaderos deben estar dispuestos a aplicar criterios técnicos y económicos efectivos para lograr un mejoramiento de los indicadores productivos y económicos obtenidos.

Grupo 4: El comportamiento productivo de este grupo de gestión arrojó resultados muy favorables para esta finca, con valores de 6,66 litros de leche por cada vaca de ordeño al día, comprendido entre 5, 0 Lt/NO/día y 7,8 LTNO/día reportados por Urdaneta et al. (1995) para fincas de la Zona Sur del Lago de Maracaibo, y similar al reportado por Peña (1995) de 6,36 Lts/VO/día para un grupo de fincas clasificadas en un nivel gerencial medio.
La productividad de leche por hectárea es de 1.678 litros, pero presenta la productividad de carne por hectárea más baja de la muestra, con un valor de 128,36 kilogramos; en tanto, sustenta una carga animal de 1,53 UAB/ha. Estos resultados sugieren un manejo animal y de potreros adecuado mediante la aplicación efectiva de insumos tecnológicos y la presencia de animales de mediano potencial lechero.

En relación a los indicadores económicos, se observa un costo de Bs. 400,01 por litro de leche. El costo por hectárea es de Bs. 671.289, el más alto de toda la muestra, y mayor que el ingreso por hectárea que alcanzó un valor de Bs. 622.415 , lo que indica un déficit por hectárea para esta finca de Bs. 48.874 y dista de su comportamiento productivo.

Los valores obtenidos para las funciones que componen el índice de gestión de este grupo, sugieren una gestión eficiente de la actividad productiva de este negocio agrícola, caracterizada por la capacidad del productor para la toma de decisiones, de manera de aprovechar los recursos disponibles para lograr los objetivos establecidos. Sin embargo, el productor-gerente de este grupo se inclinó por las funciones de organización y control, destacadas con los mayores valores (Tabla 7), lo cual supone que se parte de una planificación que parece no estar relacionada con la fase de organización; es por ello, que el productor debe determinar los recursos que posee para lograr una combinación efectiva de los mismos y a su vez resultados favorables de su actividad productiva.

Asimismo, se estima que las labores ejecutadas constituyen los medios 
para que el trabajo sea eficiente y coordinado. Además, se lleva control y evaluación del proceso de producción para alcanzar los objetivos establecidos; lo cual se considera no resulta suficientemente efectivo, ya que al tratar de obtener índices de productividad satisfactorios, este productor incurre en costos muy elevados. Esto refleja que los productores no deben intentar un incremento de su productividad mediante la utilización de tecnología sin criterios técnicos y económicos, sino mantener un equilibrio entre lo que se produce y lo que se consume para producirlo.

\section{Conclusiones}

Los resultados del estudio referente a la gerencia y productividad de los sistemas ganaderos de doble propósito en el Municipio Sucre del Estado Zulia permitieron precisar que el proceso gerencial de estas fincas carece de lineamientos empresariales formalmente establecidos.

Existen notorias deficiencias en cuanto a los criterios empresariales para definir la misión, visión, logros y objetivos, no se disponen de cronogramas de actividades ni registros adecuados, lo cual no permite el eficiente desarrollo de las operaciones y lleva a una toma de decisiones poco acertada. Asimismo, se aprecia ausencia de departamentalización y recursos humanos calificados contratados en forma oral y dificultad de los productores en la delegación de autoridad.

Estos aspectos denotan desconocimiento de criterios gerenciales por parte de los productores, lo cual repercute en la productividad, rentabilidad y competiti- vidad de las empresas ganaderas; sin embargo, los productores están conscientes de que la intuición y los criterios tradicionales por sí solos no son suficientes para gestionar eficientemente sus fincas, pues se requiere formación y capacitación para lograrlo, ya que una gerencia bien fundamentada permite alcanzar resultados satisfactorios.

Por otra parte, los resultados de los grupos de gestión definidos en esta investigación en función de la similitud en las funciones gerenciales demuestran que la manera de gestionar cada una de las actividades requeridas en una finca influye directamente en los resultados productivos y económicos de la misma; puesto que las cifras obtenidas por el grupo de gestión 1 revelan fallas en la planificación y evaluación del desempeño de estas fincas, por el contrario sugieren mayor interés por la organización, liderazgo y control del proceso productivo; este último resulta poco efectivo dados los bajos resultados productivos alcanzados por estas fincas que reflejan un manejo deficiente de los pastos y resultados económicos no satisfactorios por sus altos costos.

Contrario a este grupo, los gerentes del grupo de gestión 2 muestran el cumplimiento promedio de las funciones gerenciales obteniendo los mejores resultados productivos y económicos, lo que indica una gerencia llevada a cabo con criterios técnicos y económicos tradicionales fundamentada en la experiencia de los productores.

Los resultados del grupo 3 sugieren que estos productores realizan prácticas gerenciales poco efectivas aún cuando indican una tendencia ha- 
Gerencia y Productividad en Sistemas Ganaderos de Doble Propósito Nava Rosillón, Marbelis; Urdaneta, Fátima y Casanova, Angel

cia el manejo de sus fincas con criterio empresarial; no obstante, sus resultados productivos se consideran satisfactorios pero sus resultados económicos denotan deficiencias dadas sus bajas cifras, ya que generalmente los productores realizan mayor esfuerzo para obtener mejores beneficios y niveles de productividad.

Por su parte la finca que conforma el grupo de gestión 4 muestra elevados valores para las funciones gerenciales pero parece que la práctica no resulta suficientemente efectiva, ya que para obtener una productividad satisfactoria incurrió en altos costos disminuyendo la ganancia por hectárea de superficie.

Los sistemas ganaderos de doble propósito constituyen una actividad que implica la práctica de acciones relacionadas con la planificación, organización, liderazgo, control y evaluación de todas las operaciones requeridas para el proceso productivo que estos realizan, donde los resultados productivos y económicos indican la efectividad con la que se están ejecutando dichas acciones.

Así, en este estudio los indicadores productivos y económicos reflejan que los mejores resultados se asocian con prácticas proporcionadas de las funciones de gerencia fundamentadas en la experiencia e intuición de los productores, utilizando criterios técnicos y económicos que permitan una eficiente combinación de los factores de producción y el desarrollo de un proceso de toma de decisiones efectivo.

Lo planteado anteriormente evidencia la relación tan estrecha que existe entre la gerencia y la productividad en una empresa agropecuaria, ya que sí la práctica gerencial es poco eficiente; es decir que sí las funciones de gerencia no se cumplen a cabalidad, entonces el desarrollo de la empresa se verá afectado y los objetivos de los productores, que generalmente se establecen en función de los niveles de productividad y rentabilidad, no serán logrados; lo cual resulta contraproducente para el crecimiento y permanencia de los sistemas ganaderos en el mercado.

Por ello, los empresarios del agro deben visualizar su finca como una empresa, manejarla con criterios gerenciales y estar abiertos al cambio; pues es la forma de obtener los mejores resultados productivos, económicos y financieros convirtiéndola en una empresa agropecuaria más competitiva, rentable y exitosa.

No obstante, para que esto suceda es indispensable ofrecer formación y capacitación a los productores para alcanzar una gestión eficiente de sus fincas de manera que estas se mantengan en el tiempo, mediante la orientación y asesorías gerenciales, capacitación y adiestramiento de la mano de obra e incentivando investigaciones tanto a nivel de regiones como de municipios que contribuyan a solventar los problemas existentes en el sector agropecuario.

\section{Referencias Bibliográficas}

Bermúdez B. Alexander y Aguirre, Janet (1998). Gerencia y Aspectos Técnico -Económicos de los Sistemas de Ganadería de Doble Propósito. En: Carlos González-Stagnaro (Ed.). Mejora de la Ganadería Mestiza de Doble Propósito. Maracaibo. Venezuela. Editorial Astro Data. 643-658 pp. 
David, Fred R. (1997). Conceptos de Administración Estratégica. ( $5^{\underline{a}}$ ed.). México. Editorial Pearson Educación.

Gómez Ceja, Guillermo (1994). Planeación y Organización de Empresas. ( $8^{a} \mathrm{ed}$.). México. McGraw Hill.

Gómez Rondón, Francisco (1994). Contabilidad Ganadera en Venezuela. Caracas. Venezuela. Ediciones Fragor.

González, Baldomero (1992). Ganadería Mestiza a Base de Pastos en Condiciones Húmedas y Subhúmedas de la Cuenca del Lago de Maracaibo. En: Carlos González-Stagnaro (Ed.). Ganadería Mestiza de Doble Propósito. Maracaibo, Venezuela. Editorial Astro Data. 367-379 pp.

Guerra, Guillermo (1992). Manual de Administración de Empresas Agropecuarias. ( $2^{\mathrm{a}}$ ed.). San José. Costa Rica. Instituto Interamericano de Cooperación para la Agricultura.

Ivancevich, Jhon; Lorenzi Peter; Skinner Steven; Crosby Philip (1997). Gestión, Calidad y Competitividad. España. Editorial McGraw Hill.

Koontz, Harold y O’Donnell Cyril (1998). Administración. Una perspectiva Global. ( $6^{\text {ta }}$ ed.). México. McGraw Hill.

Morillo, Francisco y Urdaneta, Fátima (1998). Sistemas de producción con bovinos para los trópicos americanos. En: Memorias de la Conferencia Internacional sobre la Ganadería en los Trópicos. Gainesville. Florida pp: 80-104.

Ortega, Leonardo, Albornoz, Albenis y Segovia, Emma (2007). "Índice de Productividad Total de la Ganadería de Doble Propósito del Municipio Colón, Estado
Zulia - Venezuela". Revista Científica. Junio. Vol. 17. № 3. 268-274 pp.

Pacheco, Juan C.; Castañeda Widberto y Caicedo Carlos (2002). Indicadores Integrales de Gestión. Colombia. Editorial McGraw Hill.

Peña Marcano, María Elena (1995). Nivel Gerencial y Tipificación de los Sistemas de Producción de Ganadería Bovina de Doble Propósito en los Municipios Rosario y Machiques de Perijá, Estado Zulia. Maracaibo. Venezuela. Trabajo de Grado. La Universidad del Zulia. Facultad de Agronomía. Postgrado en Gerencia de Agrosistemas de Producción.

Rincón Rincón, Orlando (2004). Caracterización Técnica, Económica y Financiera de los Sistemas de Producción Bovina de Doble Propósito en la Parroquia Encontrados del Estado Zulia. San Cristóbal. Venezuela. Trabajo de Grado. Universidad Nacional Experimental del Táchira. Maestría en Gerencia de Empresas Agrícolas.

Robbins, Stephen y Coulter, Mary (1996). Administración. (5⿳亠丷厂 ed.). Traducción: Lcdo. Manuel Ortiz Satines. México. Editorial Prentince Hall.

Urdaneta, Fátima; Martínez, Edgar; Delgado Haydee; Chirinos, Zuleima; Osuna, Douglas; Ortega, Leonardo (1995). "Caracterización de los Sistemas de Producción de Ganadería de Doble Propósito de la Cuenca del Lago de Maracaibo". En: Carlos GonzálezStagnaro (Ed.). Manejo de la Ganadería Mestiza de Doble Propósito. Maracaibo. Venezuela. Ediciones Astro Data. 21-42 pp. 